\title{
Numerical experiment on the 2D cutting- stock algorithms based on local optimization
}

\author{
T. Sakamoto \\ Okayama Prefectural University \\ Soja, Okayama 719-11 Japan \\ $T E L+81-866-94-2089$ \\ FAX +81-866-94-2199 \\ E-mail sakamoto@c.oka-pu.ac.jp
}

\begin{abstract}
A numerical experiment on the computational complexity and the efficiency of layout is reported for the 2D cutting-stock algorithms based on loctl optimization. The time and the space complexity are respectively found to be $O\left(n^{2}\right)$ and better than $O\left(e_{0}\right)$ by the regression analysis, where $n$ is the number of rectangular parts and $e_{0}$ is the edge length of square sheets. The efficiency of layout is also discussed with respect to the aspect ratio of rectangular sheets.
\end{abstract}

\section{Keywords}

Cutting-stock algorithms, computational complexity, local optimization, one-to-one mapping, merge operation

\section{INTRODUCTION}

In the first paper (Sakamoto, 1993), we have proposed the six kinds of 2D cutting-stock algorithms based on local optimization. These approximate algorithms require no combinatorial process for the rectangular parts in the traditional ones, but can realize the efficiency of layout comparable to that by the dynamic programming (Adamowicz, 1976).

In this paper, we report the result of the numerical experiment on the computational complexity and the efficiency of layout of these six algorithms.

\section{OUTLINE OF THE ALGORITHMS}

Figure 1 illustrates the flow of the algorithms. The algorithms are constructed recursively using the four operations, search, divide, merge and stack. Both the shapes of parts and regions are assumed rectangular. The search operation is defined as the one-to-one mapping $f: P \mapsto W$, where $P$ and $W$ are the sets of parts $p$ and partial regions $w$ generated by the divide operation, 
respectively. A stack is used to store the data of $w$. The merge operation is used to generate a larger region using those in the stack. Figure 2 illustrates an example of the merge operation in the Cartesian coordinates. In this example, a part $p$ is allocated in the region $w$ after the search operation. The residual area of $w$ is divided into the three partial regions $w_{A}, w_{B}$ and $w_{C}$. These regions are stored in the stack and used in the subsequent search operation. The order of search for the partial regions is assumed as $w_{A}, w_{B}, M\left(w_{A}, w_{C}\right)$ and $M\left(w_{B}, w_{C}\right)$, where $M\left(w_{A}, w_{C}\right)$ (or $\left.M\left(w_{B}, w_{C}\right)\right)$ denotes the region generated by the merge operation with $w_{A}$ (or $w_{B}$ ) and $w_{C}$. The types of merge operation is classified into four due to the geometrical condition of $p$ and $w$ (Sakamoto, 1993). The local optimization used in this paper means the determination of minimizing the cost function defined by $C(p, w)=e_{x} e_{y}-c_{x} c_{y}$. By modifying the cost function, other approximate solutions can be obtained.

The significance of merge operation is explained as follows: suppose the result of search for the region $w_{A}$ (or $w_{B}$ ) is successful. Without the merge operation, the regions $w_{A}$ (or $w_{B}$ ) and $w_{C}$ are separately used in the later search operation, so that a part $p^{\prime}$ larger than these regions would not be allocated. However, since $w_{C}$ shares an edge with $w_{A}$ (or $w_{B}$ ), the region $M\left(w_{A}, w_{C}\right)$ (or $M\left(w_{B}, w_{C}\right)$ ) generated by the merge operation becomes obviously larger as long as the area of $w_{C}$ is not zero, so that the possibility for allocating $p^{\prime}$ would increase.

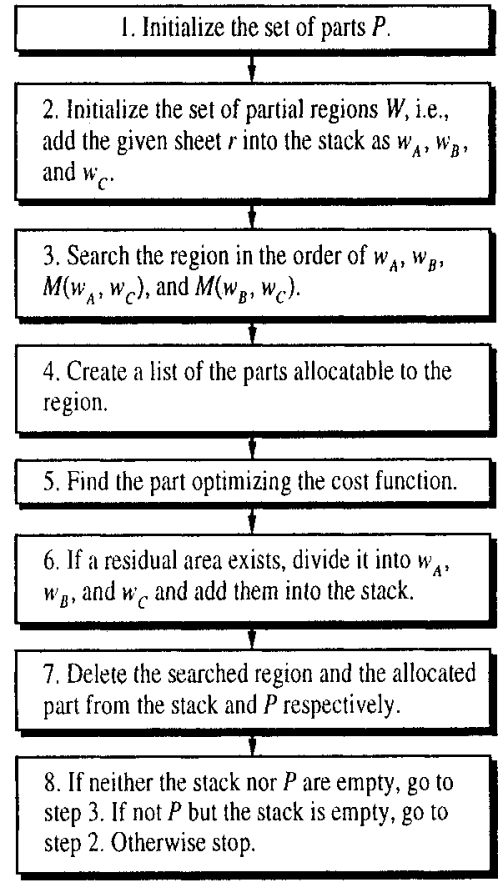

Figure 1 Flow of the algorithms.

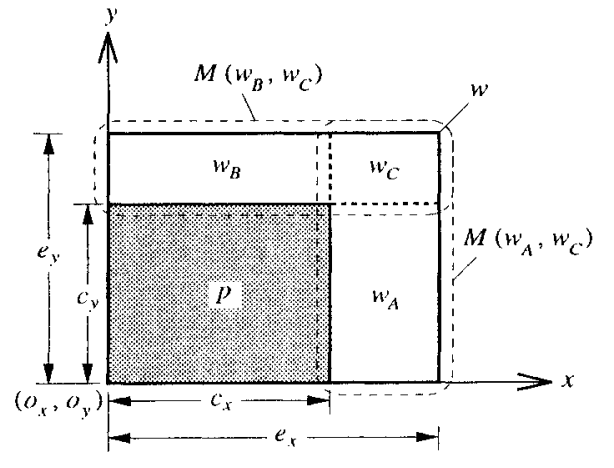

Figure 2 Conceptual diagram of merge operation in Cartesian coordinate. 
Table 1 The six algorithms considered in this paper

\begin{tabular}{ccc}
\hline Algorithrs & Orientation of the parts & Search order of regions \\
\hline A1 & region-matched & horizontal \\
A3 & region-matched & vertical \\
A4 & horizontal & horizontal \\
A5 & horizontal & vertical \\
A6 & vertical & horizontal \\
\hline
\end{tabular}

In the stack operation, it suffices in the decision of the possibility for the merge operation to check the three consecutive addresses from the memory pointer immediately after the divide operation, not the entire data in the stack. When the search and the divide operations are executed recursively, a larger region, in general, has a larger possibility to be stacked in the lower part and the search operation is applied with a priority for the smaller region. In other words, the small region, which has a smaller possibility of being allocated, has a shorter-period stay in the memory. Thus, using a stack as a memory of partial regions is effective in improving the efficiency of the data management.

In the search operation, the following two ambiguities exist;

(1) orientation of parts,

(2) order of search for partial regions.

Therefore, several kinds of algorithms can be constructed. Table I shows the six algorithms considered in this paper.

Figure 3 illustrates a layout example by the algorithm A2. The degree of freedom of parts in allocation is assumed two, i.e. either vertical or horizontal placement is possible. (a) shows the initial state. A square with unit area $(1 \times 1)$ is used as the reference. The set of sheets $R$ is composed of a single rectangular region $r(5 \times 4)$, i.e., $R=W$. Similarly the set of parts $P$ is composed of the five rectangular parts $p_{1}(2 \times 3), p_{2}(1 \times 3), p_{3}(1 \times 4), p_{4}(1 \times 1)$, and $p_{5}(2 \times 1)$. The initial state of the stack is set at $w_{C}=(5,0,0,4), w_{B}=(5,0,0,0)$, and $w_{A}=(0,0,5,4)$, where the content of the stack denotes $\left(o_{x}, o_{y}, e_{x}, e_{y}\right)$. (b) shows the placement of $p_{1}$ into $w_{A}$. First, the set $\Pi=\left\{p_{1}, p_{2}, p_{3}, p_{4}, p_{5}\right\}$ is created as the list of parts allocatable to the region $w_{A}$. Then $f\left(p_{1}\right)=(0,0,3,2)$ is determined as the mapping minimizing the cost function $C\left(p, w_{A}\right)$ for $p \in \Pi$, where the mapping $f$ denotes $\left(o_{x}, o_{y}, c_{x}, c_{y}\right)$ and $p_{1}$ is rotated. The excess region is divided into $w_{A_{1}}, w_{B_{1}}$, and $w_{C_{1}}$. After removing $p_{1}$ and $w_{A}$ from $P$ and $W$ respectively, the data of these three subregions are stored into the stack. (c) shows the result where the same operation as in (b) is iterated for three times. Namely, $f\left(p_{2}\right)=(0,2,3,1)$ is first determined for $w_{A_{1}}$. Then 


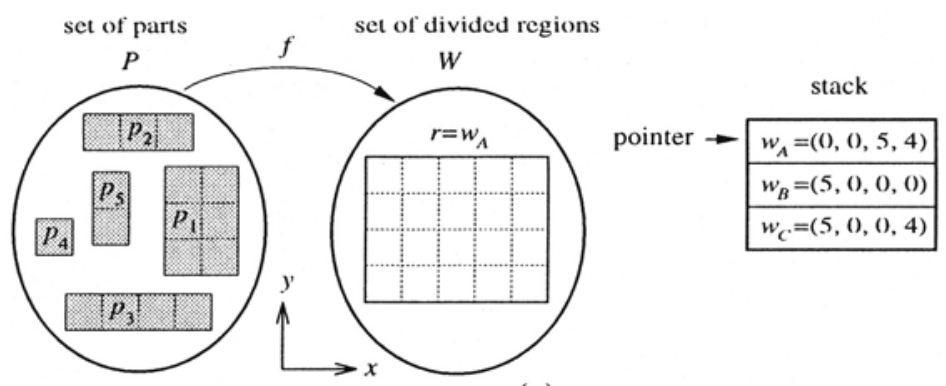

(a)
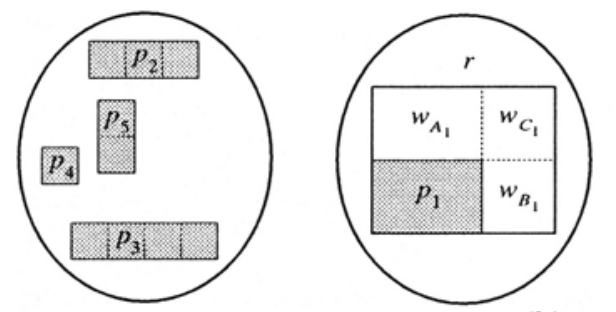

\begin{tabular}{|l|}
\hline$w_{A_{1}}=(0,2,3,2)$ \\
\hline$w_{B_{1}}=(3,0,2,2)$ \\
\hline$w_{C_{1}}=(3,2,2,2)$ \\
\hline
\end{tabular}

(b)
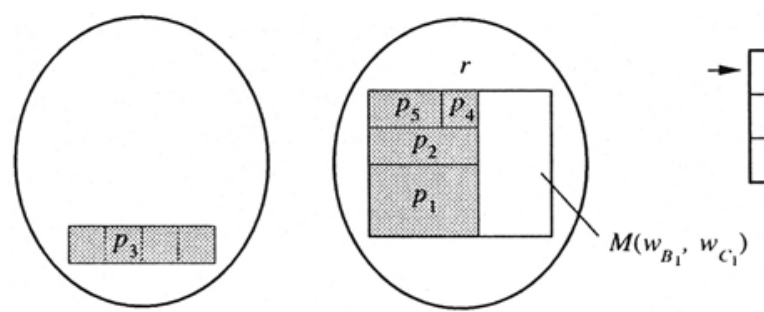

(c)
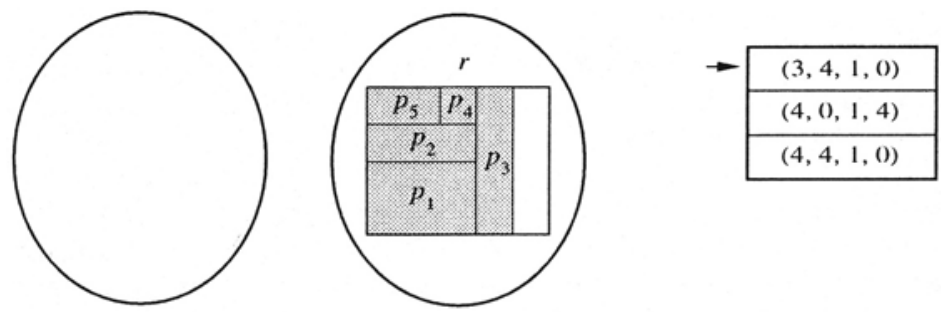

(d)

Figure 3 A layout example by the algorithm A2. (a) The initial state, (b) The result of search operation for $w_{A}$, (c) The result of the series of search operations for $w_{A_{1}}$, (d) The result of search operation for $M\left(w_{B_{1}}, w_{C_{1}}\right)$. 
$f\left(p_{5}\right)=(0,3,2,1)$ and $f\left(p_{4}\right)=(2,3,1,1)$ are determined for $w_{A_{2}}$ and $w_{A_{3}}$ respectively. When $f\left(p_{2}\right)$ is determined, $w_{B_{1}}$ and $w_{C_{1}}$ are stored in the stack as $M\left(w_{B_{1}}, w_{C_{1}}\right)$ by the merge operation for the later search operation. (d) shows the result where $f\left(p_{3}\right)=(3,0,1,4)$ is determined for $M\left(w_{B_{1}}, w_{C_{1}}\right)$. When $p_{3}$ is removed from $P, P=\phi$ holds and the algorithm stops, where $\phi$ denotes an empty set.

\section{NUMERICAL EXPERIMENT AND DISCUSSION}

Table 2 shows the data of parts used in the numerical experiment (Sakamoto, 1993), (Adamowicz, 1976). The degree of freedom of parts in allocation is assumed two. When the total number of parts is changed, the proportion of each number of parts is kept constant. The size of sheet, i.e., the initial region is $12030 \mathrm{~mm} \times 2550 \mathrm{~mm}$. When the aspect ratio of sheet $\gamma$ is changed, the area is also kept constant.

Figure 4 shows the time complexity. By the regression analysis, the time complexity is found to be $O\left(n^{2}\right)$, where $n$ is the number of part. From the flow of the algorithms, this result can be explained as follows: in the search operation, all the parts not allocated yet are tested if they can be allocated in a given region. The amount of those parts is $c_{1} n$ with $0 \leq c_{1} \leq 1$. When several parts are found as a candidate to be allocated, the optimal part is decided among those parts be allocated in a given region. The amount of those parts is $c_{1} n$ with $0 \leq c_{1} \leq 1$. When several parts are found as a candidate to be allocated, the optimal part is decided among those parts based on the criterion of local optimization. The amount of those parts is $c_{2} n$ with $0 \leq c_{2} \leq c_{1}$. Therefore, the total time complexity is $c_{1} n \times c_{2} n=O\left(n^{2}\right)$.

Table 2 The data of parts used in the numerical experiment (Sakamoto, 1993), (Adamowicz, 1976)

\begin{tabular}{crrr}
\hline \multirow{2}{*}{ Part No. } & \multirow{2}{*}{ No. } & \multicolumn{2}{c}{ Edge length } \\
\cline { 3 - 4 } & & $c_{x}(\mathrm{~mm})$ & $c_{y}(\mathrm{~mm})$ \\
\hline 1 & 96 & 450 & 270 \\
2 & 48 & 400 & 270 \\
3 & 16 & 1140 & 170 \\
4 & 8 & 2500 & 300 \\
5 & 8 & 2500 & 139 \\
6 & 40 & 300 & 270 \\
7 & 32 & 1500 & 270 \\
8 & 32 & 270 & 110 \\
9 & 8 & 1993 & 165 \\
10 & 16 & 710 & 675 \\
11 & 16 & 500 & 500 \\
12 & 32 & 800 & 500 \\
13 & 30 & 300 & 74 \\
14 & 8 & 1360 & 160 \\
15 & 8 & 1760 & 785 \\
\hline
\end{tabular}




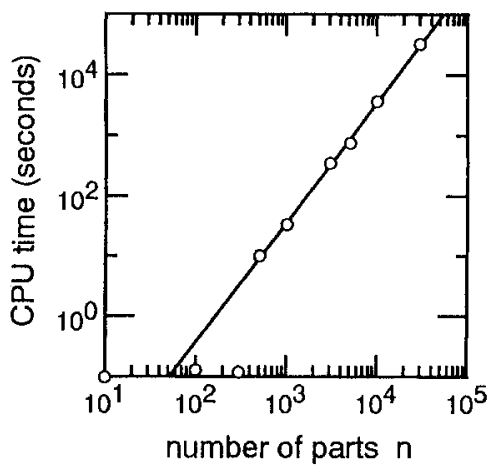

Figure 4 Time complexity.

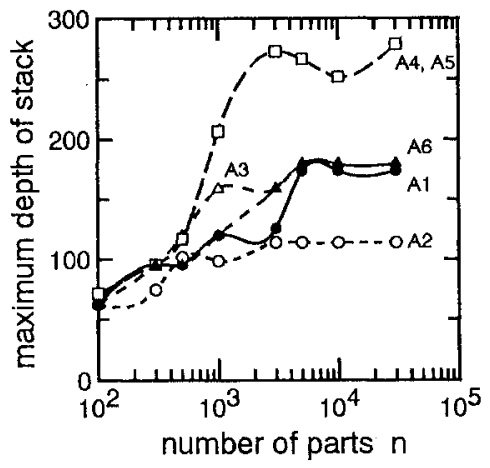

Figure 5 Dependence of the maximum depth of the stack on the number of parts $n$.

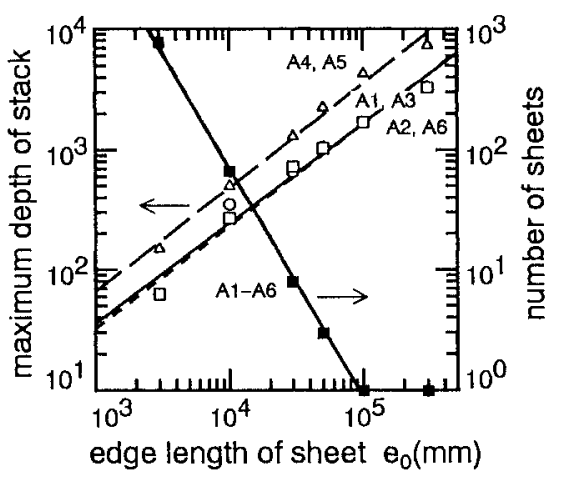

Figure 6 Space complexity at $n=30000$ and $\gamma=1$.

Since the size of stack cannot be predicted, the maximum depth of stack is used as a measure for the space complexity. Figure 5 shows thedependence of the maximum depth of the stack on the number of parts $n$. It appears that the maximum depth tends to saturate as increasing $n$ and is almost constant at $n=30000$. Thus, to analyze the space complexity, $n$ and $\gamma$ are respectively set at 30000 and one. Figure 6 shows the result. The space complexity is found to be better than $O\left(e_{0}\right)$ by the regression analysis, where $e_{0}$ is the edge length of the sheet. Also the required number of sheets is $O\left(e_{0}{ }^{-2}\right)$ as is expected because the total area of parts is kept constant. Figure 7 shows the efficiency of layout $\eta$, where $\eta$ is defined as the ratio of the total area of parts and that of sheets. (a) shows the influence of $n$ with $\gamma=1 . \eta$ varies seriously among the algorithms for smaller $n$, but converges as increasing $n$. The required number of sheet is nearly proportional to $n$. (b) shows the influence of $\gamma$ with $n=30000$. Also $\eta$ varies seriously among the algorithms as $\gamma$ departs from one, but there exists a pair of algorithms whose $\eta$ 's are symmetric around $\gamma=1$. At this aspect ratio, every algorithm generates a similar highest $\eta$. 


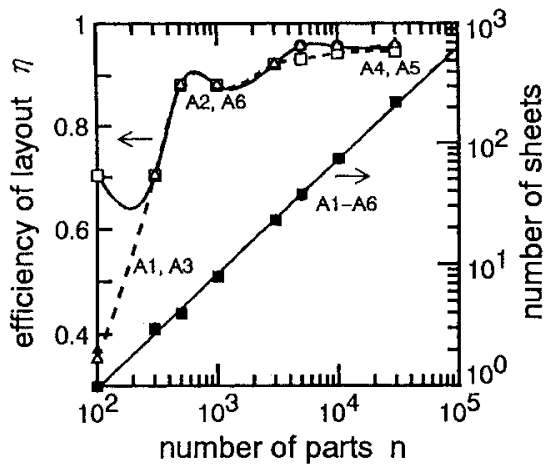

(a)

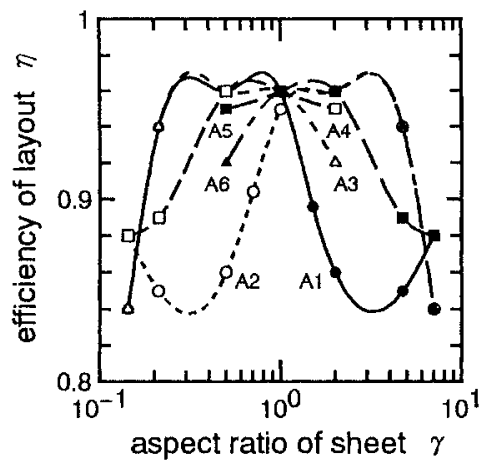

(b)

Figure 7 Efficiency of layout. (a) dependence on the number of parts, $\gamma=1$, (b) dependence on the aspect ratio of sheet, $n=30000$.

\section{SUMMARY}

A numerical experiment on the computational complexity and the efficiency of layout has been reported for the six kinds of 2D cutting-stock algorithms based on local optimization. The time and the space complexity are respectively found to be $O\left(n^{2}\right)$ and better than $O\left(e_{0}\right)$ by the regression analysis, where $n$ is the number of part and $e_{0}$ is the edge length of the rectangular sheet.

\section{REFERENCES}

Sakamoto, T. (1993) 2D cutting-stock algorithms based on local optimization, Trans. IEICE Japan, 76-A, 605-11.

Adamowicz, M. and Albano, A. (1976) A solution of the rectangular cutting-stock problem, IEEE Trans. Syst. Man Cyber., SMC-6, 302-10. 